\title{
Chiral nematic liquid crystals as an alternative filling in photonic crystal fibers
}

\author{
Marzena M. Tefelska, ${ }^{* 1}$ Tomasz R. Woliński, ${ }^{1}$ Roman Dąbrowski, ${ }^{2}$ and Jan Wójcik ${ }^{3}$ \\ ${ }^{I}$ Faculty of Physics, Warsaw University of Technology, Koszykowa 75, 00-662 Warszawa, \\ ${ }^{2}$ Military University of Technology, Warszawa, Poland \\ ${ }^{3}$ Maria Curie Sklodowska University, Lublin, Poland
}

Received March 15, 2010; accepted March 29, 2010; published March 31, 2010

\begin{abstract}
Chiral nematic liquid crystals (LCs) are very interesting materials to infiltrate photonic crystal fibers since they are characterized by unique optical properties such as selective Bragg reflection, circular dichroism and optical activity. In this letter first experimental results of chiral nematic LCs-infiltrated photonic crystal fibers are presented.
\end{abstract}

Photonic crystal fibers (PCFs) are a new class of optical fibers which have attracted significant attention for the last few years [1,2]. The propagation of light in these structures can be guided by two different mechanisms: index guiding and the photonic bandgap effect (PBG). One of the ways to modify the properties of PCFs is to fill their holes with various materials. LCs are very interesting materials to infiltrate PCFs [3-9] and especially chiral nematic liquid crystals due to their spatial periodicity are characterized by unique optical properties such as selective Bragg reflection, circular dichroism, and optical activity. This phase exhibits helicoidal structure, i.e. spatial twisting of molecules perpendicular to the director. The helical pitch $p$ is a very important parameter that characterizes chiral nematic LCs and is defined as a distance it takes for the director to rotate one full turn in the helix. The helix can be righthanded or left-handed and this property can be used to construct chiral nematic LCs-based circular polarizers.

In this letter preliminary experimental results of photonic crystal fibers filled with chiral nematic liquid crystals are presented. The PW500 chiral nematic liquid crystal mixture used in the experiment was composed of alkylcyclohexyl and bicyclohexylbenzene nitryles and isothiocyanates and an optically active dopant (OAD) (10\% weight content). The chemical structure of the OAD is shown in Fig. 1. The PW500 is characterized by significantly reduced temperature sensitivity of the selective Bragg reflection, see Fig.2. Investigations of other physical parameters of the PW500 chiral nematic mixture are still in progress.

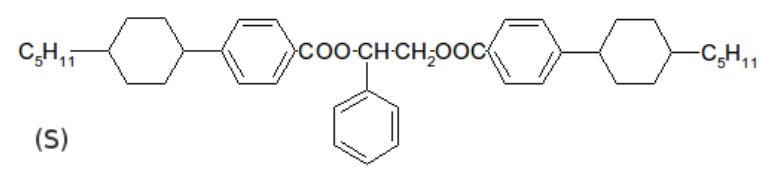

Fig. 1. Chemical structure of the OAD of the PW500 mixture

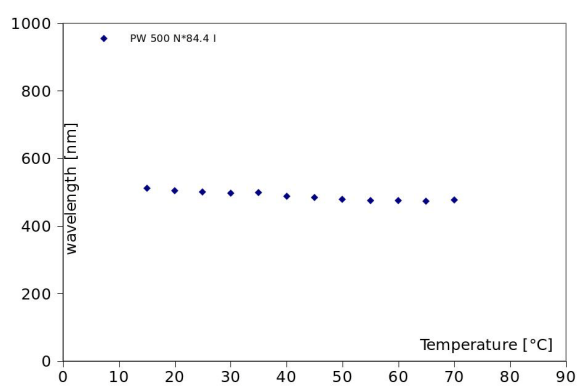

Fig. 2. The selective Bragg reflection for the PW500 chiral nematic LC

As a host two PCFs were used. PCFs with 3 and 5 rings of holes were manufactured at the Maria Curie Sklodowska University (UMCS, Lublin, Poland) (Fig 3.). The $070124 \mathrm{PCF}$ with 3 rings has a $3.9 \mu \mathrm{m}$ core diameter, whereas the LMA 081120 PCF with 5 rings has the fiber and core diameters of $\sim 130 \mu \mathrm{m}$ and $3.2 \mu \mathrm{m}$, respectively.
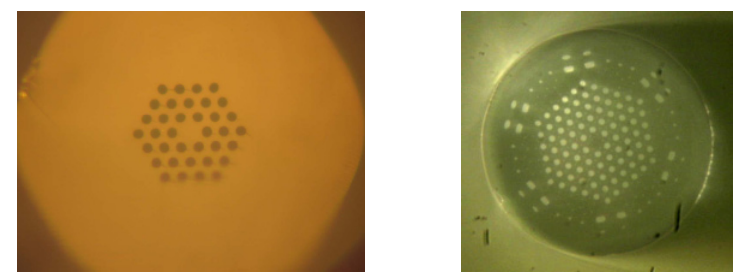

Fig. 3. Cross-sections of the photonic crystal fibers with a) 3 rings (070124) and b) 5 rings (LMA 081120) of holes.

\footnotetext{
*E-mail: a.author@if.pw.edu.pl
} 
The experimental setup consists of a halogen lamp as a light source, a Peltier module to control temperature in the range from $-10^{\circ} \mathrm{C}$ to $100^{\circ} \mathrm{C}$ and a HR4000 Ocean Optics spectrometer used as a detector.

PBGs tuning in the PCFs filled with a chiral nematic LC was observed. Initially, the photonic crystal fiber with 3 rings of holes was filled with the PW500 mixture in a $2.5 \mathrm{~cm}$ PCF section. We noticed that photonic band gaps were moving into shorter wavelengths when the temperature was increasing. The temperature was changed from $20^{\circ} \mathrm{C}$ to $72^{\circ} \mathrm{C}$ and from $-10^{\circ} \mathrm{C}$ to $20^{\circ} \mathrm{C}$ (Fig. 4, 5). As it can be seen from Fig. 6, the power of light for the PCF with 3 rings of holes filled with the PW500 was relatively high in a wide temperature range. The highest peak in Fig. 6 (at $72^{\circ} \mathrm{C}$ ) was obtained in the isotropic phase of the PW500 chiral nematic LC.

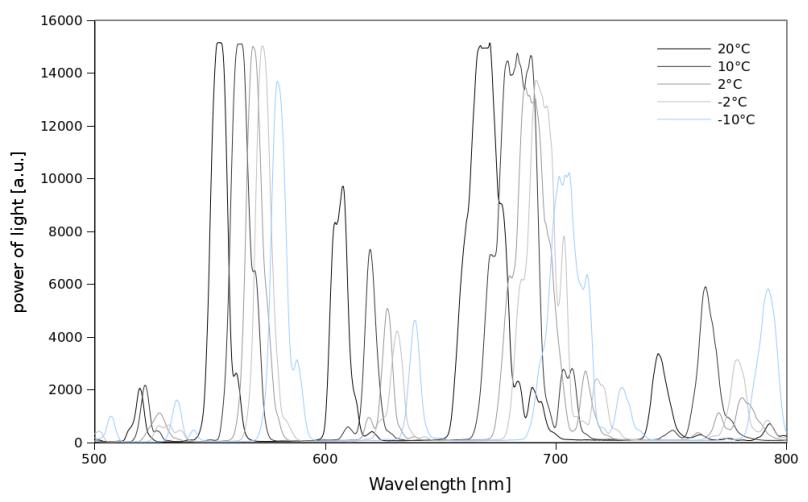

Fig. 4. PBGs are moving into shorter wavelengths under the influence of temperature from $-10^{\circ} \mathrm{C}$ to $20^{\circ} \mathrm{C}$

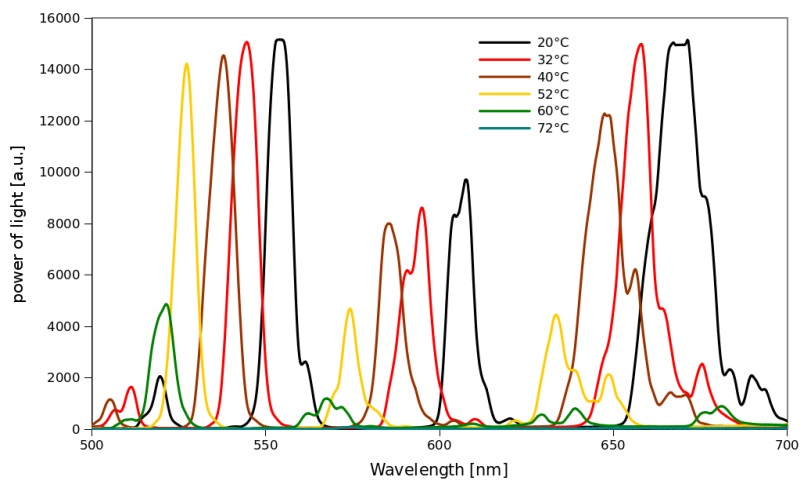

Fig. 5. PBGs are moving into shorter wavelengths under the influence of increasing temperature from $20^{\circ} \mathrm{C}$ to $72^{\circ} \mathrm{C}$.

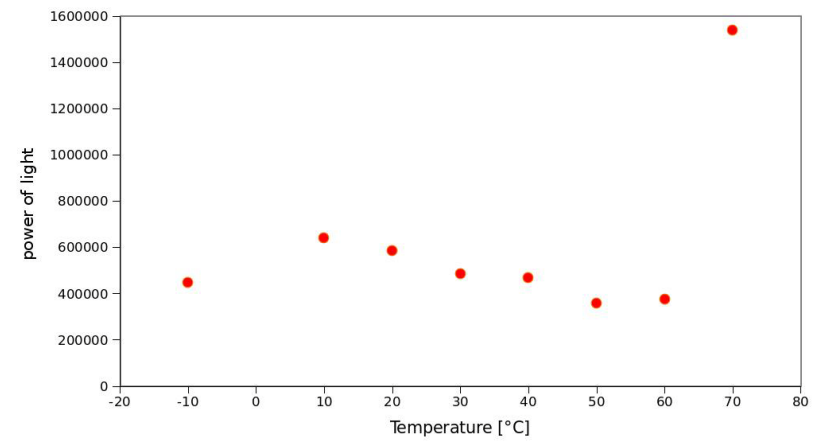

Fig. 6. The power of light under the influence of temperature for the PCF with 3 rings of holes filled with the PW500, for the halogen lamp spectrum.

Similar experimental results were observed for the PCF with 5 rings of holes filled with the PW500 mixture. PBGs tuning under the influence of temperature was observed. The section of LC infiltration was about $6.4 \mathrm{~cm}$ long. The PCF with the PW500 mixture was cut to smaller sections up to $0.5 \mathrm{~cm}$ to calculate the attenuation of light (Fig.7). For the $6.4 \mathrm{~cm}$ long section with the PW500 filling light attenuation was relatively high and in particular for the wavelengths $435 \mathrm{~nm}$ and $630 \mathrm{~nm}$, residual propagation was observed. The longer infiltration lengths of the chiral nematic LC caused attenuation in the LC-infiltrated PCF to be higher (Fig.8). Attenuation was calculated by using the formula (1):

$$
A=\frac{1}{x} \cdot 10 \log \left(\frac{P(x)}{P_{i}}\right)
$$

Where $A$ - attenuation, $x$ - length of the PCF section filled with the LC, $P(x)$ - output power, $P_{i}$ - input power.

Fig. 9 presents the attenuation of a PCF with 5 rings of holes with the PW500 chiral nematic LC for different filling lengths. We estimated the averaged attenuation of the fiber at the level $\sim 5.5 \mathrm{~dB} / \mathrm{cm}$. This value is about 1000 times higher than the attenuation of an empty PCF, which was measured as $\sim 0.005 \mathrm{~dB} / \mathrm{cm}$. The experimental values were measured at room temperature. 


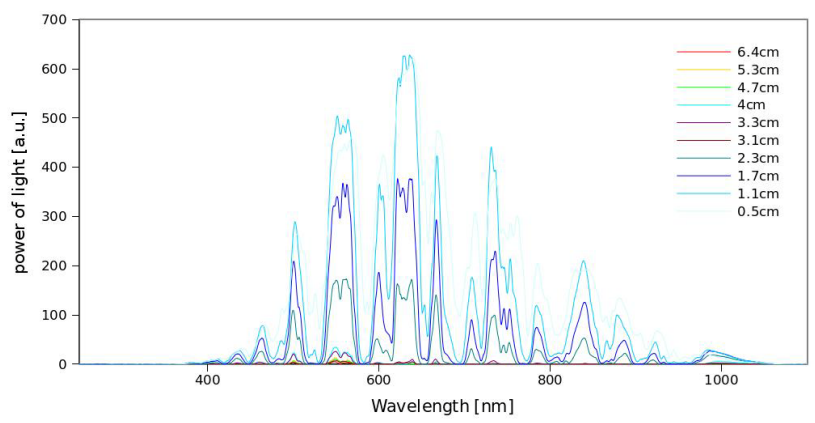

Fig. 7. Spectrum of the light propagating in the PCF with 5 rings of holes with PW500 for different filling lengths.

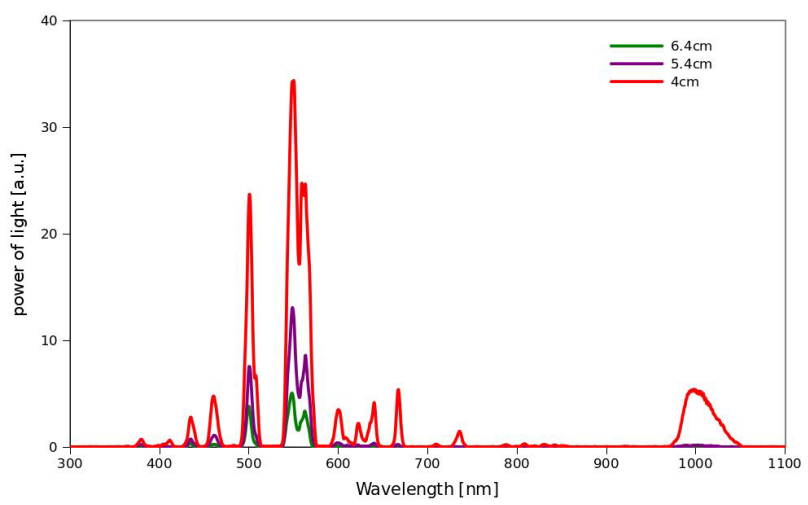

Fig. 8. Spectrum of the light propagating in the PCF with 5 rings of holes with the PW500 for different filling lengths.

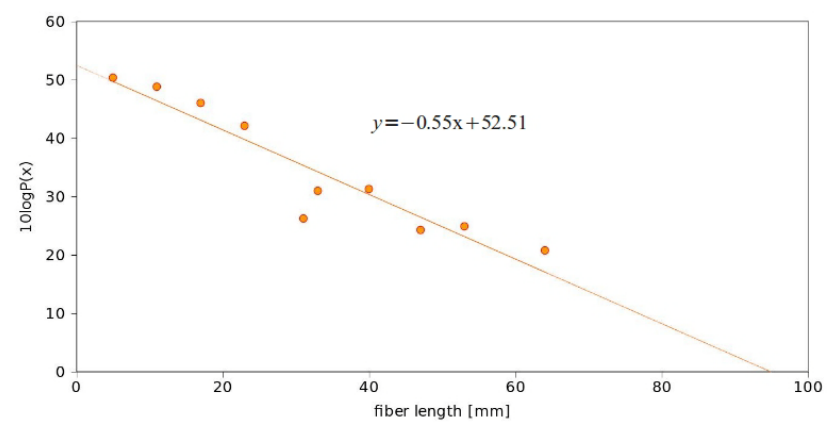

Fig. 9. Power of light of the PCF with 5 rings of the holes with the PW500 chiral nematic LC for different filling lengths.
In comparison to Fig. 4, 5 in Fig. 7, 8 we used a different PCF sample (host structure and its length) and also different coupling conditions.

It was observed that there is no direct relation between the reduced thermal sensitivity of the PW500 mixture and spectral properties of the infiltrated PCF.

The configuration of a PCF with 3 rings of holes filled with the PW500 mixture demonstrates the possibility of optical filtering by temperature-induced tuning of the photonic band gaps. The attenuation in the temperature range from $-10^{\circ} \mathrm{C}$ to $60^{\circ} \mathrm{C}$ is very low. Consequently, this property may be used to propose a new idea of a fiber-optic filter.

This work was supported by the Polish Ministry of Science and Education under the grant N517 056535. One of the authors (M. Tefelska) acknowledges the European Social Fund through the Warsaw University of Technology Development Programme sponsored by the European Union.

\section{References}

[1] P. St. J. Russell „Review: Photonic Crystal Fibers” Science 299, 358-362 (2003).

[2] C. Knight, "Photonic crystal fibres", Nature 424, 847-851 (2003).

[3] F. Du, Y. Q. Lu, and S. T. Wu, Electrically tunable liquid-crystal photonic crystal fiber, Appl. Phys. Lett. 85, 2181-2183 (2004).

[4] T. Alkeskjold, J. Lægsgaard, A. Bjarklev, D. Hermann, A. Anawati, J. Broeng, J. Li, and S.-T. Wu, All-optical modulation in dye-doped nematic liquid crystal photonic bandgap fibers. Opt. Express 12, 5857-5871 (2004).

[5] T. R. Woliński et al., Meas. Sci. Technol. 17, 985-991 (2006)

[6] T.T. Larsen, A. Bjarklev, D.S. Hermann, and J. Broeng, "Optical devices based on liquid crystal photonic bandgap", Opt. Express 11, 2589-2596 (2003).

[7] T. R. Woliński, S. Ertman, P. Lesiak, A. W. Domański, A. Czapla, R. Dąbrowski, E. Nowinowski-Kruszelnicki, and J. Wójcik, „Photonic liquid crystal fibers - a new challenge for fiber optics and liquid crystals photonics", Opto-Electronics Review 14, 329334, (2006).

[8] T. R. Woliński, A. Czapla, S. Ertman, M.Tefelska, A. W. Domański, E. Nowinowski-Kruszelnicki, R. Dąbrowski, „Tunable highly birefringent solid-core photonic liquid crystal fibers", Opt. Quantum Electron. 39, 1021-1032 (2007).

[9] T.R. Wolinski, K. Szaniawska, S. Ertman, P. Lesiak, A. W. Domanski, R. Dabrowski, E. Nowinowski-Kruszelnicki and J. Wojcik "Influence of temperature and electrical fields on propagation properties of photonic liquid-crystal fibres", Meas. Sci. Technol. 17, 985-991 (2006). 